\title{
Unexpected Massive Hemorrhage Following the Removal of a Pleural Drainage Tube
}

\author{
Minako Seki ${ }^{1}$ and Satoshi Yoda ${ }^{2}$
}

\begin{abstract}
We herein describe a case in which a massive hemorrhage unexpectedly occurred after the removal of a pleural drainage tube which had been in place for five days. One possible explanation for that event was the damage of the intercostal artery during tube insertion into the thoracic cavity. This is an extremely rare but severe complication. Therefore, the present report provides useful information for physicians who treat patients with respiratory diseases.
\end{abstract}

Key words: pleural tube drainage, removal, iatrogenic hemothorax

(Intern Med 54: 953-954, 2015)

(DOI: 10.2169/internalmedicine.54.3719)

\section{Case Report}

An 85-year-old Japanese man was admitted to our hospital to receive treatment for right pleural effusion following the onset of bacterial pneumonia. His stature was $161.7 \mathrm{~cm}$, body weight $77.5 \mathrm{~kg}$, and the laboratory tests indicated mild leukocytosis (white blood cell count: 11,100/ $\mathrm{L}$ ) and elevated serum C-reactive protein $(17.82 \mathrm{mg} / \mathrm{dL})$ and fibrin degradation product $(555.1 \mathrm{mg} / \mathrm{dL})$ levels, which were attributed to the patient's pleuritis. As the patient had a history of cerebral infarction, he had been taking aspirin (100 $\mathrm{mg}$ /day), but no coagulation disorders were detected by the laboratory tests [prothrombin time-second (PT-S) $12.1 \mathrm{sec}$, prothrombin time-percent (PT-P) $98.3 \%$, prothrombin timeinternational normalized ratio (PT-INR) 1.01 and activated partial thromboplastin time (APTT) $28 \mathrm{sec}$. On days 1 and 10 of the patient's hospital stay, thoracentesis was performed to drain the remaining fluid. Part of the effusion was sampled for bacterial examinations and bacteria was not detected. Despite antibiotic therapy, chest X-rays demonstrated that the pleural effusion continued to increase in volume. On day 12 , tube drainage was performed by an experienced respiratory physician. An $8 \mathrm{Fr}$ drainage kit (comprising a soft tube made by urethane resin and a needle as an inner cylinder) was uneventfully inserted via the right side of the pa- tient's back under local anesthesia and ultrasonographic guidance. About $720 \mathrm{~mL}$ of sero-bloody pleural effusion were drained immediately, and smaller amounts of fluid were discharged on the following days. The laboratory tests following tube drainage did not indicate anemia during pleural drainage. After 5 days, the drainage tube was removed. However, a pulsatile hemorrhage immediately developed at the drainage site and a large hematoma rapidly appeared on the patient's back. Although the hemorrhage was easily controlled by suturing, the patient complained of discomfort and immediately fell into a state of shock. He required a rapid drip infusion and had to remain in the head-down position in order to stabilize his blood pressure. An emergent chest X-ray examination and blood sampling was performed, and the patient was diagnosed with hemorrhagic shock due to a massive hemothorax (Fig. 1, 2).

An emergency operation was scheduled to both confirm the location of the bleeding and remove the intrathoracic hematoma, and a blood transfusion was performed while the patient was waiting for the operation. The operation was performed with the patient in the lateral decubitus position using a thoracoscope. The continuous bleeding was controlled prior to thoracoscope insertion, however, many clots surrounded the drainage site. Although there were no obvious findings indicative of a lacerated vessel, we considered the drainage tube to most likely have been the responsible

${ }^{1}$ Department of Surgery, Japan Community Health Care Organization (JCHO), Saitama Medical Center, Japan and ${ }^{2}$ Department of Internal Medicine, Japan Community Health Care Organization (JCHO), Saitama Medical Center, Japan

Received for publication July 22, 2014; Accepted for publication September 4, 2014

Correspondence to Dr. Minako Seki, lazarj250@live.jp 


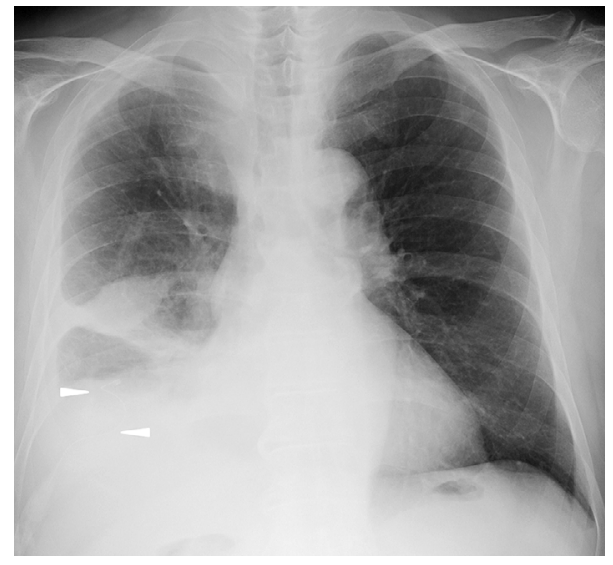

Figure 1. Chest X-ray obtained from the patient in the standing position before tube removal. The arrows indicate the drainage tube.

cause for the massive bleeding.

Approximately 1,200 mL of clotted blood had accumulated in the patient's pleural cavity. After the removal of the blood by aspiration, the pleural cavity was washed with saline. Then, the intercostal tissues (including the muscles and small branch of vessels around the extubation site) were subjected to electrical ablation and clipping. Finally, two pleural drainage tubes were inserted via the port sites and the operation was completed. The patient's postoperative hospital stay was uneventful, and he was discharged from the Department of Surgery on the 4th postoperative day.

\section{Discussion}

Several severe complications related to pleural drainage have been reported $(1,2)$, most of which occur during or following insertion of a drainage tube or needle. Postthoracentesis pneumothorax is the most common of these complications, occurring in $3-30 \%$ of patients (1-3). Other complications, such as hemothorax caused by arterial injuries (3), direct injuries to intrathoracic or intraabdominal organs (4) (e.g., the lungs, liver, and spleen), vasovagal reflex $(1,2)$, and systemic hypotension due to pain during the insertion procedure, have also been reported. Reexpansion pulmonary edema is also a relatively common complication and may cause severe respiratory distress after pleural drainage.

While it is known that physicians must be careful to prevent air inhalation into the thoracic cavity during chest tube removal, severe hemorrhagic complications following drainage tube removal or pulsatile bleeding due to injury to any artery of the thoracic wall (most likely the intercostal artery) have not been reported. In the present case, we believe that the tube damaged the intercostal artery during its insertion into the thoracic cavity, but then compressed the wound site

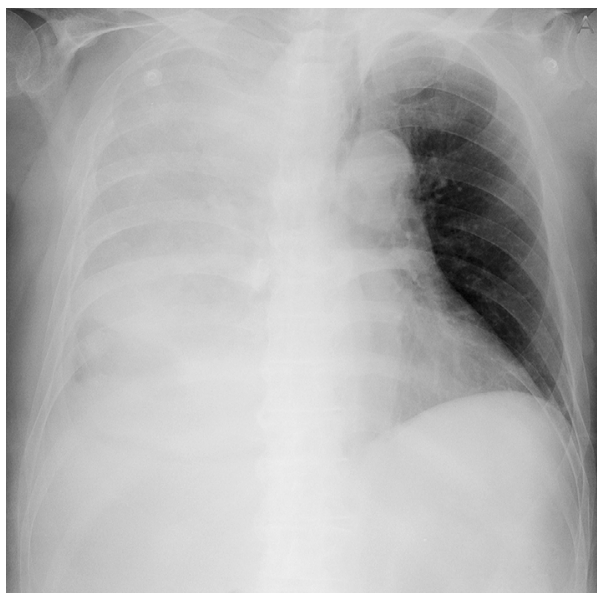

Figure 2. Chest X-ray obtained from the patient in the supine position after tube removal.

during the pleural drainage, preventing massive bleeding. Therefore, when the tube was removed bleeding may have occurred from the previously compressed site or the wound was re-opened. This adverse event may be related to the patient's advanced age, inflammatory state, and/or history of aspirin intake. As the location of the bleeding could not be confirmed intraoperatively, the above explanation is speculative. However, it was clear that the removal of the tube had thus caused the adverse event. Many reports have so far discussed safety management during drainage tube insertion (2); for example, the usefulness of ultrasonographyguided procedures has been well documented (5). However, massive hemorrhaging associated with drainage tube removal is difficult to predict. Therefore, physicians must be aware that severe hemothorax can be caused during both the insertion and removal of drainage tubes.

The authors state that they have no Conflict of Interest (COI).

\section{References}

1. Collins TR, Sahn SA. Thoracocentesis: clinical value, complications, technical problems, and patient experience. Chest 91: 817822, 1987.

2. Jones PW, Moyers JP, Rogers JT, Rodriguez RM, Lee YC, Light RW. Ultrasound-guided thoracentesis: is it a safer method? Chest 123: 418-423, 2003.

3. Yacovone ML, Kartan R, Bautista M. Intercostal artery laceration following thoracocentesis. Respir Care 55: 1495-1498, 2010.

4. Haron H, Rashid NA, Dimon MZ, et al. Chest tube injury to left ventricle: complication or negligence? Ann Thorac Surg 90: 308309, 2010.

5. Duncan DR, Morgenthaler TI, Ryu JH, Daniels CE. Reducing iatrogenic risk in thoracentesis: establishing best practice via experimental training in a zero-risk environment. Chest 135: 1315-1320, 2009.

(C) 2015 The Japanese Society of Internal Medicine http://www.naika.or.jp/imonline/index.html 